Chair of Comparative Public Policy and Administration Department of Politics and Management University of Konstanz

\title{
Politikbeendigung
}

\section{als policyanalytisches Konzept}

Michael W. Bauer

Universität

Konstanz

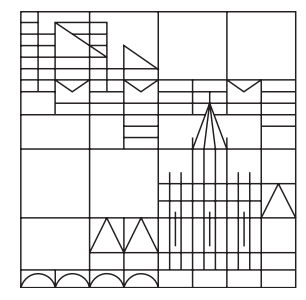




\section{Chair of Comparative Public Policy and Administration Department of Politics and Management \\ University of Konstanz}

Working Paper 02/2006

\section{Politikbeendigung als policyanalytisches Konzept}

Michael W. Bauer

Also publ. as:

Bauer, Michael W.: Politikbeendigung als policyanalytisches Konzept,

in: Politische Vierteljahresschrift, 47 (2006), 2, S. 147-168

Konstanzer Online-Publikations-System (KOPS)

URL: http://www.ub.uni-konstanz.de/kops/volltexte/2008/6326/

URN: http://nbn-resolving.de/urn:nbn:de:bsz:352-opus-63260

\section{Contact}

Prof. Dr. Christoph Knill

Chair of Comparative Public Policy

and Administration

Box D 91

D-78457 Konstanz

Germany

Phone ++497531 885597

Fax ++ 497531882381

christoph.knill@uni-konstanz.de

University of Konstanz

www.uni-konstanz.de

Department of Politics and Management

www.uni-konstanz.de/sektionen/polver

Chair of Comparative Public Policy and Administration www.uni-konstanz.de/FuF/Verwiss/knill 


\title{
Politikbeendigung als policyanalytisches Konzept
}

\author{
Dr. Michael W. Bauer, \\ Wissenschaftlicher Assistent, \\ Lehrstuhl für vergleichende Policy-Forschung und Verwaltungswissenschaft, \\ Fachbereich Politik- und Verwaltungswissenschaft, \\ Universität Konstanz
}

\begin{abstract}
Der Aufsatz analysiert die policyanalytische Literatur zum Konzept der Politikbeendigung (Policy Termination). Die seit der Entwicklung des Konzepts vorgenommenen Revisionen werden ebenso behandelt wie die nach wie vor bestehenden Defizite dieser Forschungsrichtung. Angesichts vermehrter Hinweise auf die in jüngster Zeit steigende Anzahl von Beendigungsphänomenen in westlichen Wohlfahrtstaaten und insbesondere in Deutschland werden empirische sowie methodisch-konzeptionelle Perspektiven aufgezeigt, wie das Konzept der Politikbeendigung für die aktuelle Policyforschung gerade in theoriebildender Absicht wieder fruchtbar gemacht werden kann.
\end{abstract}

\section{Einführung}

Sind öffentliche Politiken gegen ihre Beendigung in einem besonderen Maße gefeit? Welches sind die Faktoren, die eine Politikbeendigung erleichtern oder systematisch erschweren? Was bedeutet es für ein demokratisches System im Allgemeinen und für das Steuerungspotential des öffentlichen Sektors im Besonderen, wenn der Abbau einmal installierter Programme ein mühsames politisches Unterfangen darstellt, dem zudem in der Regel wenig Aussicht auf Erfolg beschieden ist? Das sind einige der zentralen Fragen, die aus policyanalytischer Perspektive erstmals Mitte der 1970er Jahre aufgeworfen wurden. ${ }^{1}$ Die sich anschließenden Debatten um Begriffe, Konzepte und um die Theoretisierung der "Policy Termination” oder „Politikbeendigung“ stehen im Mittelpunkt dieses Aufsatzes. ${ }^{2}$

Der Impuls, die systematische Beschäftigung mit der Politikbeendigung auf die Forschungsagenda zu setzen, kam Anfang der 70er Jahre von Garry D. Brewer. Brewer ging es vor allem darum, das von seinem Lehrer Harold D. Lasswell

\footnotetext{
${ }^{1}$ Es existiert neben der Beendigungsperspektive auf Politiken auch eine ausgiebige Literatur, die sich der Auflösung von Organisationen, den Lebenszyklen von Organisationen und der Expansion bzw. Kontraktion von Organisationspopulationen widmet (vgl. Kaufmann 1976, 1987; Hannan/Freeman 1977; 1985; Levine 1978; Carroll 1983, 1984; Kimberly 1981; Whetten 1987; Hood/Dunsire 1981; Norris-Tirell 1997, 2001; Brown 1997; Lewis 2002; Corder 2004; Kuipers/Boin 2005). Diese umfangreiche Literatur kann in diesem Aufsatz nur insoweit erörtert werden, wie es für das Verständnis der Politikbeendigung als policyanalytisches Konzept notwendig ist.

${ }^{2}$ Ich benutze in dieser Arbeit den Begriff Policy Termination überwiegend um in theoriegeschichtlicher Perspektive den Gegenstand der amerikanisch dominierten Debatte zu kennzeichnen, ansonsten wird der eingedeutschte Begriff Politikbeendigung bevorzugt.
} 
angestoßene Projekt der „Policy Science” zu vervollständigen und fest in der amerikanischen Forschungslandschaft zu verankern. Sich in dieser Situation der Politikbeendigung als der „missing link“33 zuzuwenden, schien damals opportun. Denn die Politikbeendigung war bis dato als einzige Phase in dem von Lasswell entwickelten Modell ${ }^{4}$ des Politikphasenzyklus weder empirisch noch theoretisch ausgiebig behandelt worden. Vor dem Hintergrund des mittlerweile längst aufgegebenen Anspruchs der Policy Science, den Phasenzyklus als rationales Projekt politischer Problemverarbeitung und -lösung zu konzipieren, konnte zudem die aus steuerungstheoretischer Sicht unerlässliche Beendigung erfolgloser Politiken nicht auf Dauer außen vor bleiben. ${ }^{5}$

Die ersten Jahre der Terminierungsforschung waren äußerst ertragreich. ${ }^{6}$ In kurzer Abfolge erschienen bis zum Ende des Jahrzehnts eine Reihe von Politikbeendigungsstudien, von denen retrospektiv allerdings nur Behn (1978), Brewer (1978) und insbesondere deLeon (1978) größere Bedeutung zukommt. In den 1980er Jahren nahmen sich nur noch vereinzelt Veröffentlichungen dem Thema der Politikbeendigung an. Ab Mitte der 1990er Jahre kam es dann wieder zu einer kleineren Terminierungskonjunktur, die auf niedrigem Niveau bis zur Gegenwart anhält (Sato 2002). Sie wurde 1997 von einem Sonderheft des International Journals of Public Administration unter der Herausgeberschaft von Mark D. Daniels eingeläutet (Daniels 1997; deLeon 1997; Frantz 1997; Greenwood 1997; Harris 1997; Norris-Tirrell 1997) und hatte ihren letzten Höhepunkt in einem ebenfalls von Daniels geleiteten Symposium in der gleichen Zeitschrift (Daniels 2001; Geva-May

\footnotetext{
${ }^{3}$ „Although implementation has been described as the "missing link" in policy analysis, termination clearly has been a less attended issue" (deLeon 1978b: 369). Peter deLeon, von dem die These der Policy Termination als der "eigentlichen missing link" stammt, bezieht sich im angeführten Zitat insbesondere auf Hargrove 1975.

${ }^{4}$ Garry D. Brewer geht von den Phasen Initiation/Invention, Estimation, Selection, Implementation, Evaluation und schließlich Termination aus (vgl. Brewer 1974).

${ }^{5}$ Als Garry D. Brewer 1974 die Herausgeberschaft der einschlägigen Fachzeitschrift „Policy Sciences” übernahm, räumte er dem Thema Politikbeendigung als einer „phase ... whose importance in current affairs demands attention" auch sogleich hohe Priorität ein (Brewer 1974: 241). Bereits zwei Jahre später erscheint ein Sonderheft der Zeitschrift, in dem sich eine Reihe von Autoren mit der Vorstellung erster Fallstudien der Beendigungsthematik annehmen (Behn 1976; Biller 1976; Bradley 1976; Foster/Brewer 1976; Iklé 1976; Lambright/Sapolsky 1976; Shulsky 1976; Wallerstein 1976). Eugene Bardach fungiert als Herausgeber dieses Sonderheftes. Seine auf der Grundlage der Fallstudien basierende Auswertung (Bardach 1976) zusammen mit der zeitgleich andernorts erscheinenden, äußerst einflussreichen Studie von Herbert Kaufman, in der dieser der Frage nachgeht, „Are Government Organizations Immortal?" (Kaufman 1976), bilden bis heute zentrale Referenzpunkte der Politikbeendigungsdebatte.
} 
2001; deLeon/Hernández-Quezada 2001; Norris-Tirrell 2001; Harris 2001).

Dabei ist anzumerken, dass Politikbeendigung als policyanalytisches Konzept in der europäischen Politikwissenschaft nicht weiter rezipiert worden ist. Policy Termination blieb allerdings auch in den USA - gerade im Vergleich zu den einflussreichen Forschungsanstrengungen in den verwandten Bereichen der Implementation und Evaluation öffentlicher Programme (Mayntz 1983; Sabatier 1993a; Wollmann 2003) eher ein Nebenschauplatz.

Diese Vernachlässigung des Studiums der Politikbeendigung überrascht insofern als in Zeiten staatsbudgetärer Turbulenzen und der zunehmenden Beschwörung eines bedrohlichen Verlusts der politischen Steuerbarkeit des Gemeinwesens (auch) aufgrund eines perzepierten Unvermögens, Staatsaufgaben auf ein finanzierbares Maß zurückzuführen, das Wissen um die Beendigungsbedingungen öffentlicher Interventionen eigentlich Hochkonjunktur haben müsste (Biller 1976; Daniels 2001; BertelsmannStiftung 2005). Die Vernachlässigung erstaunt aber auch aus der Perspektive einer auf Verallgemeinerung abzielenden empirischen Policy Analyse. Denn eine zeitgemäße Policy Analyse dürfte an der Untersuchung des angenommenen Zusammenhangs zwischen steigender Steuerungsunfähigkeit und erfolglosem Aufgabenabbau einerseits sowie der systematischen Einordnung des Phänomens (erfolgreicher oder verhinderter) Politikbeendigung andererseits schon deshalb interessiert sein, weil sie sonst den Anspruch aufgeben müsste, zentrales Analyseinstrument für ein umfassendes Verständnis des Politikprozesses zu sein.

Vor diesem Hintergrund führt der Artikel in die Konzepte der Politikbeendigung ein (Abschnitt 2) und erläutert die wichtigsten Revisionen (Abschnitt 3). Anschließend werden die nach wie vor bestehenden Defizite benannt (Abschnitt 4). Im Mittelpunkt steht die Frage, ob es Erfolg versprechende Ansätze gibt, das policyanalytische Beendigungskonzept zur Erklärung aktueller Politikprozesse fruchtbar zu machen (Frantz 2002, 2003; Geva-May 2004). Abschnitt 5 skizziert die theoretischen und methodischen Anforderungen für ein solches Unternehmen, ohne diese jedoch im Rahmen dieses Artikels auch empirisch umsetzen zu können. Abschnitt 6 will einen Beitrag zur Einlösung der Forderung nach einer Erneuerung des

\footnotetext{
${ }^{6}$ Von den insgesamt ungefähr 50 wissenschaftlichen Beiträgen, die zum Kern dieses Forschungsunternehmens gerechnet werden können (vgl. Literaturverzeichnis), stammt etwa ein Viertel aus dieser ersten Forschungswelle.
} 
Politikbeendigungsansatzes leisten und entwickelt Vorschläge zur Differenzierung des Konzepts sowie zur weiteren Hypothesenbildung. Der Aufsatz endet mit einem zusammenfassenden Ausblick, in dem auch die Frage nach der Aktualität der Beendigungsproblematik öffentlicher Politiken behandelt wird (Abschnitt 7).

\section{Die Grundlegung des Terminierungsansatzes}

Obwohl die Politikbeendigung bereits früh als besondere Phase in der Policy Science Literatur auftaucht, ist es das Verdienst von Eugene Bardach auf der Grundlage der von ihm edierten Fallstudien erste systematische Schlussfolgerungen gezogen und entsprechende Forschungshypothesen formuliert zu haben. Bardach versteht Policy Termination als politischen Prozess, in dessen Mittelpunkt die politische Auseinandersetzung steht, ähnlich wie bei der Phase der Politikformulierung. Politikbeendigung ist demnach „a special case of the policy adoption process: there is a struggle to adopt a policy $A$, the substance of which is to eliminate policy $B^{\prime \prime}$ (Bardach 1976: 126). Allerdings sei bei der Politikbeendigung ganz besonders mit „vested interests” zu rechnen, da diese mitunter machtvolle moralische Argumente über die Ungerechtigkeit und Unfairness von Terminierungsbeschlüssen vorbringen können. "The most interesting theoretical aspect of termination politics is the interrelationship between the political order and the moral order. It is this connection that gives struggles over policy termination a rather special cast when compared with more run-of-the-mill battles over public policy. It also helps to explain why policy termination battles are particularly hard to win, in fact, that they are very infrequently even attempted“ (Bardach 1976: 127). Das geringe Interesse der Sozialwissenschaft erklärt Bardach damit, dass wegen des seltenen Auftretens des Phänomens eine pessimistische Einschätzung der Generalisierungsmöglichkeiten unter Wissenschaftlern vorherrsche. ${ }^{7}$ Gerade aber in Zeiten, in denen breit angelegte staatliche Interventionen immer weniger als Erfolg versprechend angesehen werden,

\footnotetext{
7 „A ... reason for the scholarly neglect of this subject is that it is hard to study such an infrequent phenomenon as policy termination. One always suspects that each instance of the phenomenon is bound to be so idiosyncratic that no interesting generalizations will be possible. Since social scienceand social scientists-thrive on generalizations rather than idiosyncracies, termination has never become ,hot' as a topic of academic interest“ (Bardach 1976: 123).
} 
sei es „precisely the rarity of the phenomenon that makes it important“ (Bardach 1976: 123). Terminierung selbst würde, wenn sie denn vorkomme, entweder als „Glockenschlag“ („bang“) oder als ein sehr langes „Wimmern” („a very long whimper“) auftreten. Bardach sieht insbesondere fünf Faktoren, die Terminierung erschweren: Erstens müssten erhebliche „sunk costs” in Gestalt rechtlicher Normsetzung und bestehender budgetärer Abwicklungsmechanismen abgeschrieben werden. Zweitens schlage die Konfliktstruktur besonders „brutal“ zu Buche, und Politiker würden es daher zu vermeiden suchen, vorsätzlich den Widerstand von Bürgern auf sich zu ziehen, denen etwas genommen werden soll, woran sie bislang gewohnt waren. Dabei kommt Koalitionen der Terminierungsgegner - zumeist Programmbegünstige, private Zulieferer und der die öffentliche Aufgabe tragende Personalkörper - ganz besondere Bedeutung zu. Drittens würden Politiker auch deshalb nur ungern mit Terminierungsbeschlüssen in Verbindung gebracht werden, weil die Gefahr bestünde, mit dem Argument konfrontiert zu werden, "If it's that bad as you and others now say, why did you permit it to go on for so long?" Viertens sei es auch verständlich, dass Politiker und Manager davor zurückschreckten, den existierenden Organisationskörper zu beschädigen oder Personal zu verunsichern, wenn sie selbst damit rechnen müssen, auf deren Loyalität und Funktionsfähigkeit einmal angewiesen zu sein, beispielsweise wenn es nämlich darum geht, eigene Programmprojekte künftig erfolgreich umzusetzen. Ein letzter Grund für die Scheu vor Politikbeendigung ist in der allgemeinen Ausrichtung des gesellschaftlichen Erfolgskriteriums zu sehen. „The American political system, like most others, rewards novelty and innovation“ (Bardach 1976: 129). Auf der anderen Seite können auch Faktoren ausgemacht werden, die die Wahrscheinlichkeit der Politikbeendigung erhöhen: erstens ein Regierungswechsel; zweitens Veränderungen in der ideologischen Großwetterlage und eine daraus gegebenenfalls resultierende Delegitimierung einer öffentlichen Aufgabe. Drittens „a period of turbulence“, worunter Bardach eine Zeit versteht, in der düstere Erwartungen über die gesellschaftliche Entwicklungen vorherrschen, die die Menschen psychologisch bereits darauf einstimmen, dass sich die Zukunftsaussichten ohne schmerzhafte Einschnitte weiter verschlechtern werden. Viertens kann sich die Abfederung der Auswirkungen einer Terminierungsentscheidung (großzügige Übergangsregelungen, Beschäftigungsgarantie für betroffenes Personal usw.) positiv auf Beendigungsvorgänge auswirken. Und schließlich könne weitsichtiges Politikdesign 
(im Sinne von Zero-Base-Budgeting oder Sunsetregelungen) im weiteren Verlauf die Wahrscheinlichkeit einer Politikbeendigung erhöhen (Bardach 1976: 129-130; vgl. auch Biller 1976).

Ein weiterer einflussreicher Artikel stammt von Robert D. Behn „How to Terminate a Public Policy: A Dozen Hints for the Would-be Terminator" (Behn 1978a). In Form von 12 Empfehlungen ${ }^{8}$ wertet Behn darin auf ironisierende Weise seine empirischen Befunde zur Policy Termination als dem „neglected butt of the policy process“ aus (Behn 1978a: 413; vgl. ferner Behn 1976, 1977, 1978b). Dabei sollte man sich von dem leichten Tonfall der Arbeit nicht täuschen lassen, denn implizit entwickelt Behn ein Modell der Minimierung des Widerstands gegen Politikbeendigung und rückt damit die Politics Dimension radikal in den Mittelpunkt (Daniels 1997b: 59). In offensichtlicher Anlehnung an die Implementationsstudie von Pressmann und Wildavsky sieht Behn die Wahrscheinlichkeit von erfolgreicher Politikbeendigung dann erhöht, je weniger Entscheidungspunkte oder -schnittstellen den Gegnern der Beendigung zur Einflussnahme eröffnet werden und desto besser sich der Terminierungsbeschluss medienwirksam an eine ideologisch gestützte Negativbewertung der zu beendenden Politik einbetten lässt. Dabei vergisst Behn nicht zu unterstreichen, dass Policy Terminierung an sich aus polit-praktischer Perspektive wohl immer ein Minusgeschäft bleiben wird: Eine Politikbeendigung anzugehen, „...is not likely to be a very rewarding undertaking. Your chances of succeeding are poor, and even if you prevail, you will not make friends doing so" (Behn 1978a: 393). Wenn aber letztlich allein die politische Listigkeit der Akteure über den Erfolg von Politikbeendigungsbeschlüssen entscheidet, stellt sich jenseits der 12 Behn'schen Empfehlungen die grundsätzliche Frage nach der ethischen und demokratischen Legitimierbarkeit derartiger Strategien.

Gegen Ende der ersten Welle von Terminierungsstudien resümiert Garry D. Brewer,

\footnotetext{
${ }^{8}$ Ohne sie weiter auszuführen, seien diese Empfehlungen („hints“) aufgelistet: 1. Don't float trial balloons; 2. Enlarge the policy's constituency; 3. Focus attention on the policy's harm; 4 . Take advantage of ideological shifts to demonstrate harm; 5 . Inhibite compromise; 6 . Recruit an outsider as administrator/terminator; 7. Avoid legislative votes; 8. Do not encroach upon legislative prerogatives; 9. Accept short-term cost increases; 10. Buy off the beneficiaries; 11. Advocate adoption not temination; 12 . Terminate only what is necessary.
} 
der die Diskussion um die Policy Terminierung ja angestoßen hatte, das etwas zwiespältige Resultat der bisherigen Forschungsanstrengungen. Demnach sei „termination (...) frequently only the replacement of one set of expectations, rules, and practices with another. In this sense, termination signals a beginning as much as it does an end“ (Brewer 1978: 339). Im Anschluss an Brewer stellte sich Peter deLeon der Aufgabe, die bisherigen Resultate der Beendigungsdiskussion zu systematisieren und begriffliche wie theoretische Schlussfolgerungen zu ziehen (deLeon 1978a,b). DeLeons Arbeit figuriert etwas ambitioniert unter dem Titel „A Theory of Policy Termination“ (deLeon 1978a). ${ }^{9}$ Dort wird jedoch nicht die versprochene Theorie der Politikbeendigung geliefert, sondern es werden vielmehr sozusagen ex negativo - die einer Terminierung im Weg stehenden Hindernisse ansatzweise theoretisiert. Die Bestandteile seiner „Beendigungverhinderungstheorie“ sind eine klare Definition der Terminierung selbst, eine Typologie dessen, was im öffentlichen Raum überhaupt beendet werden kann, sowie die Herausarbeitung von sechs zentralen Gründen, warum Policy Termination so schwer geplant und ausgeführt werden kann. Ziel ist es dabei, die Politikbeendigung in den Kontext der anderen Phasenabschnitte zu stellen und ihre Rückwirkungen auf jene - und umgekehrt die Einflüsse des Politikzyklusprozesses auf die Politikbeendigung erfassbar zu machen. Policy Terminierung wird demgemäß als „the deliberate conclusion or cessation of specific government functions, programs, policies or organizations“ (deLeon 1978a: 280) definiert. ${ }^{10}$ Die Typologie dessen, was gemäß des „Policy“ Terminierungsbegriffs beendet werden kann, umfasst individuelle Programme, Politiken, Organisationen und Staatsfunktionen. In eben dieser Reihenfolge steigern sich auch die Terminierungshürden. Staatsfunktionen - zu denken ist etwa an Verteidigung, Aufrechterhaltung der inneren Sicherheit, materielle Umverteilung - werden de facto wohl nie beendet. Organisationen, die deLeon als „groups of individuals that constitute what we call institutions“ bzw. als eingesetzt, „to respond to a specific need“ (1978a: 284) begreift, sind bei dieser Hierarchisierung

\footnotetext{
${ }^{9}$ Dieser Artikel ist allerdings fast wortgleich mit dem anderen 1978er Artikel von deLeon zum selben Thema, den er unter dem Titel „Public Policy Termination: An End and a Beginning“ (deLeon 1978b) publizierte.

${ }^{10}$ DeLeon bietet ferner, und zwar um die Restriktivität seiner Terminierungsdefinition abzumildern, die ja auch beträchtliche Politikveränderungen nicht als Beendigung gelten lässt, noch das Konzept einer „partial termination“ an, „in which specific government functions, programs, policies or organizations significantly redirect their activities" (deLeon 1978a: 280). Da dieses Konzept nicht weiter von der Literatur aufgenommen wurde, soll es in diesem Abschnitt nicht weiter vertieft werden (vgl. aber Abschnitt 6).
} 
etwas einfacher zu beenden als die Staatsfunktionen selbst, zeichnen sich aber - mit Herbert Kaufmans Ergebnissen im Hinterkopf ${ }^{11}$ - durch große Resistenz und Langlebigkeit aus. Policies als „generalised approaches or strategies toward solving a particular problem“ (deLeon 1978a: 284) seien dagegen leichter zu beenden, da die respektiven Organisationen, die mit deren Umsetzung betraut sind, eher einzelne „Policies“ opfern als sich selbst in Terminierungsgefahr zu bringen; und, ferner, weil Organisationen in der Regel über ein breiteres Netz an Unterstützern verfügen als einzelne Policies, zumal letztere durch ihr Einwirken auf die Gesellschaft normaler Weise auch ernste Kritiker generieren. Am unsichersten ist demgemäß das Schicksal einzelner Programme, anhand derer letztlich eine Policy umgesetzt und oft auch definiert wird. „They are closest to the problem and therefore their impact can be most directly measured and, if found lacking, blame most easily affixed“ (deLeon 1978a: 285). Als die sechs zentralen Gründe für die seltene Planung und Umsetzung von Politikbeendigung identifiziert deLeon im Hauptteil seines Artikels kognitive Abneigung, institutionelle Langlebigkeit, dynamischen Konservatismus, Antiterminierungskoalitionen, rechtliche Hindernisse und hohe Anschubskosten. ${ }^{12}$ Die kognitive Abneigung bezieht sich nicht nur auf die allgemeine intellektuelle Reserviertheit gegenüber der Beendigungsthematik an sich, sondern meint insbesondere den Umstand, dass im Regelfall viele der an einem Beendigungsprozess zu beteiligenden Akteure emotionale oder professionelle Investitionen in die zu beendende Organisation oder Policy getätigt haben. Dies

\footnotetext{
${ }^{11}$ In einer einflussreichen Studie, die auch in den Kontext der frühen Policy Terminierungsdebatte einzuordnen ist, ging Herbert Kaufman (1976) der Frage nach, „are Government Organizations Immortal?" Kaufman vergleicht die Anzahl von „federal agencies“ im Jahr 1923 mit der im Jahr 1973. „There were 175 organizations in the 1923 sample. No less than 148 of them (nearly 85 percent) were still going in 1973“ (1976: 34). Demnach wurden also nur ganze 27 „agencies” aufgelöst. Ferner sind in diesen 50 Jahren 166 neue Organisationseinheiten, die bis zum Vergleichsjahr 1973 nicht aufgelöst wurden, hinzugekommen. Kaufman vergleicht die „Sterberate“ öffentlicher Organisationen mit privaten Unternehmen und kommt zu dem Schluss, dass im privaten Sektor die Terminierungswahrscheinlichkeit doppelt so hoch ist wie im öffentlichen Bereich (1976: 54). Die größten Bedrohungen gehen für die staatlichen Organisationen aus von „competition, changes in leadership and policy, obsolescence resulting from routinization and adherence to past methods, and completion of mission. ... Organizational death seems to claim victims in all age categories without systematic discrimination. There was, however, a faint tendency for the oldest organizations to fare better than their juniors, particularly the youngest" (Kaufman 1976: 60). Somit scheint Kaufman empirisch nachzuweisen, dass es tatsächlich eine beeindruckende Fähigkeit öffentlicher Einrichtungen gibt, sich zu erhalten und an veränderte Rahmenbedingungen anzupassen Die These Kaufmans galt lange Zeit als Paradigma in der Terminierungsliteratur. Sie wurde allerdings bereits früh theoretisch kritisiert und jüngere empirische Arbeiten konnten sie nicht bestätigen (vgl. Peters/Hogwood 1988, 1991; Lewis 2002).
} 
verringert jenseits rationaler Abwägungen die Motivation dieser Akteure, einen Terminierungsbeschluss mitzutragen (Biller 1976: 137). Die institutionelle Langlebigkeit als zweiter Widerstandsfaktor ist nicht weiter erstaunlich, da „policies and expecially organizations are deliberately designed to endure. ... The crucial point is not whether planned institutional longevity is good or bad, but simply that it is a fact of political, bureaucratic life" (deLeon 1978a: 288). Interessanter scheint dagegen der Faktor dynamischer Konservatismus zu sein. Damit wird der Umstand umschrieben, dass Organisationen oder Policies keine statischen Gebilde sind, sondern „dynamic entities" (deLeon 1978a: 289), die relativ einfach auf andere Ziele ausgerichtet oder in anderen Problembereichen eingesetzt werden können. Natürlich steigt die Terminierungswahrscheinlichkeit für eine Organisation oder ein Programm, die ihre Aufgaben nicht erfüllen. Aber die hinter diesen Politiken und Organisationen stehenden Akteure bleiben nicht passiv, wenn das eigene berufliche Überleben auf dem Spiel steht. Sie werden nach Wegen suchen, durch entsprechenden Wandel der Beendigungsandrohung den Wind aus den Segeln zu nehmen. Bereits an anderer Stelle ausführlich besprochen wurde die Kraft von Antiterminierungskoalitionen (Behn 1978b; Bardach 1976; Biller 1976). Unter diesen Koalitionen versteht deLeon „significant political groups that will be opposed to termination. ... Each has its own assets and tactics, but they are particularly successful when they form coalitions to block threatened termination acts" (deLeon 1978a: 290). Hierunter fallen insbesondere das begünstigte Klientel einer bestimmten Politik, die privaten "Zulieferer", die von der Produktion der öffentlichen Intervention ihren Lebensunterhalt bestreiten und die im engeren Sinne staatlichen Angestellten, deren Karriereaussichten oder sogar Beschäftigungsverhältnisse durch eine Politikbeendigung in Gefahr geraten würden. Die rechtlichen Hindernisse im Sinne von Einspruchs- und Klagemöglichkeiten der in irgendeiner Weise von einem Terminierungsbeschluss Betroffenen sollten in einem Rechtsstaat zwar nicht als etwas an sich Negatives betrachtet werden. De facto kann die Inanspruchnahme rechtlicher Überprüfung staatlicher Entscheidung in der Tat auch als Verhinderungsund Verzögerungstaktik dienen. Sollen die fünf zuvor erörterten Faktoren überwunden werden, kann das im buchstäblichen Sinne sehr teuer werden und dazu führen, dass diejenigen die sich aus Effizienzgründen für eine Politikbeendigung

12 Im Original ,intellectual reluctance', ,institutional permanence', ,dynamic conservatism', ,antitermination coalitions', ,legal obstacles' und ,high start-up costs' (vgl. deLeon 1978a: 286 ff.; vgl. 
einsetzen, mit schmerzhaft hohen „Anschubskosten“ konfrontiert werden. DeLeon beendet seinen Artikel mit einigen Ideen, wie es trotz solch kolossaler Widerstände ermöglicht werden könnte, die Chancen dafür zu verbessern, ineffektive Politiken öfter als bisher zu beenden. Er schlägt unter anderem vor, ex-ante in das Programmdesign schon "Entscheidungspunkte“ einzuplanen, an denen Terminierungsbeschlüsse getroffen werden können. Insgesamt müssten aber die Anreize so verändert werden, dass die Beteiligten Terminierung nicht als zu verhindernden Misserfolg oder gar als Existenzgefährdung begreifen, sondern als Möglichkeit zur Aufgabenoptimierung und daher als Stärkung ihrer eigenen Position im Politikprozess (deLeon 1978a: 296).

\section{Kritik und Revision}

Gegen Ende der ersten Welle der Terminierungsliteratur machte sich Enttäuschung darüber breit, dass sich die bis dato erfasste Empirie der Politikbeendigung nicht passgerecht in das steuerungstheoretisch-rationale Projekt der Policy Sciences und des Politikzyklus einfügen ließ (Brewer 1978; Cameron 1978; Bothun/Comer 1979; deLeon 1982, 1983). Die wichtigsten Revisionsvorschläge können unter den Stichworten Ideologie, Sukzession, Kosten und Anreize zusammengefaßt werden.

Ideologie. Eine erste Kritik zielte unmittelbar auf die aufklärerische Prämisse der frühen Arbeiten und rückte mit dem ideologischen Kontext der Politikbeendigung die antirationalen Elemente des Terminierungsprozesses in den Mittelpunkt. Demnach war Terminierung nie als rationales Abwägen der Akteure zu erklären, sondern der in einer Beendigungsentscheidung zum Tragen kommende Politikwandel hängt ursächlich mit Veränderungen auf der die jeweilige Politik stützenden Werteebene zusammen. Diese Kritik war in der Sache berechtigt, wenn auch etwas ungerecht. Denn die Autoren der ersten Welle hatten es in ihrem Streben, möglichst alle Einflussfaktoren im Terminierungsfeld zusammenzutragen, sträflich vernachlässigt, die gefundenen Variablen auch in eine hierarchische Ordnung zu bringen. Es war daher relativ einfach, an konkreten Fallbeispielen die Mächtigkeit von Ideen, Werten und von Weltbildern in der politischen Auseinandersetzung um Politikbeendigung als zentrale Einflussdimension festzumachen (Cameron 1978; Harris 1997). So musste 
auch Peter deLeon, der noch 1983 den Rationalisierungsgehalt von Beendigungsprozessen durch eine systematische Verknüpfung von Programmevaluierung und Terminierung gestärkt sehen wollte (deLeon 1983), zugeben, dass für die Beendigung einer Politik „economics and efficiencies ... have been much less the guiding rationale than fundamental questions of values and ideologies" (deLeon 1987: 191). ${ }^{13}$

Sukzession. Das Thema der Politikbeendigung als Ende und Anfang zugleich (Brewer 1978, deLeon 1978b) streifen Brian W. Hogwood und B. Guy Peters mit ihrem Konzept der Policy Succession. Demnach ist öffentliche Politikgestaltung ein Prozess des dynamischen Wandels und eine Beendigungsabsicht mündet in der Regel in eine veränderte bzw. angepasste Fortsetzung (Hogwood/Peters 1982, 1985; vgl. auch Grafton 1984 sowie Weaver 1988). ${ }^{14}$ Im Grunde war dieser Aspekt der Politiksukzession auch den frühen Arbeiten zur Politikbeendigung nicht verborgen geblieben. Ausgehend von der klassischen Arbeit von Edward L. Katzenbach aus dem Jahr 1958 interessierten sich die Terminierungsforscher immer auch für Aspekte der „Wiedergeburt“ oder des paradoxen Mangels an tatsächlicher Politikbeendigung (vgl. Kotz 1988). ${ }^{15}$ Ironischer Weise war es auch die äußerst plausible Rekonzeptualisierung des Politikbeendigungsprozesses als Sukzession, die verhinderte, dass in einer Zeit, die ohnehin durch eine selbstkritische Neuausrichtung der universitären Policy Forschung geprägt war (Windhoff-Héritier 1987; Héritier 1993), größere intellektuelle Anstrengungen auf dem Gebiet der Policy Termination unternommen wurden (Greenwood 1997).

Kosten. Waren die Terminierungsforscher anfangs davon ausgegangen, dass zu hohe Kosten im Vergleich zum Politikergebnis eine herausragende Rolle im Beendigungsprozess spielen (Bardach 1976; Kaufman 1976), wurde schnell deutlich,

\footnotetext{
${ }^{13}$ DeLeons Artikel von 1983 verweist auf die mangelnde Verbindung von Evaluierungsergebnissen und Politikbeendigung und damit auf ein strukturelles Defizit beider Forschungszweige. In der Tat hat auch die Evaluierungsforschung immer wieder über die Nichtbeachtung ihrer Ergebnisse im Politikprozess geklagt.

${ }^{14}$ Nach Hogwood und Peters sei die analytische Entscheidung, ein Phänomen der Policy Termination in den Untersuchungsmittelpunkt zu rücken oftmals "like talking about the death of the caterpillar without mentioning the birth of the butterfly" (1982: 227). Ebenso selten wie zu regelrechten Politikbeendigungen kommt es, nach den Autoren, allerdings zu genuinen Politikinnovationen.

15 Katzenbach (1958) untersuchte in einer Fallstudie die erstaunliche Langlebigkeit der Pferdekavallerie in der Armee der Vereinigten Staaten, obwohl der militärische Nutzen von Pferden längst durch den waffentechnologischen Fortschritt aufgehoben war. Nick Kotz (1988) zeichnet die mehrmalige Aussetzung und stetige Wiederkehr des B-1 Bomber-Projekts nach.
} 
dass Effizienz- und Effektivitätsgründe nicht wirklich im Zentrum von tatsächlichen Terminierungsbemühungen stehen. Die ersten empirischen Ergebnisse deuteten sogar darauf hin, dass erfolgreiche Beendigungen nicht selten eine (zumindest kurzfristige) Erhöhung der Kosten in Kauf nehmen müssen, um ihr Ziel zu erreichen (Behn 1978a). Diese Befunde fanden in der Folge immer wieder ihre empirische Bestätigung (Frantz 1997, 2003; deLeon 1997; Daniels 1997a, b). Demnach kann eine grundlegende Spaltung von allgemeiner politischer Rhetorik, in der sehr wohl und sehr häufig Kostenaspekte in den Vordergrund gestellt werden, und den konkreten Umständen von Politikbeendigungen konstatiert werden, bei denen die tatsächlichen Einsparergebnisse zumeist keine entscheidende Rolle spielen. Letzthin wurde allerdings versucht, mit der Rekonzeptualisierung des Kostenbegriffs - als emotionale, legitimatorische Kosten usw. - die notwendigen politischen Ressourcen im Beendigungswettstreit als verschiedene „Kostenarten“ besser unterscheidbar und so für ein differenzierteres Terminierungskonzept fruchtbar zu machen (Frantz 2002).

Anreize. Der Beginn der Terminierungsdebatte Mitte der 1970er Jahre fällt zusammen mit der Amtsübernahme von Jimmy Carter als Präsident der Vereinigten Staaten. Die Besetzung des Themas Politikbeendigung durch führende Vertreter der Disziplin wird auch damit in Zusammenhang gebracht, dass die neue CarterAdministration sich anschickte erstmals in großem Stil systematisch "Sunset"Bestimmungen in die amerikanische Programmgesetzgebung einzufügen, um den Kongress zu zwingen, nach einer bestimmten Periode und im Lichte der dann möglicher Weise vorliegenden Ergebnisse über die Weiterführung bestimmter Politiken abzustimmen. Eine ähnliche Zielrichtung verfolgte das damals kontrovers diskutierte „Zero-Base-Budgeting“, wonach jährlich „von Null an“ und nicht auf der Grundlage der Vorjahreshöhe über einzelne Budgetposten beraten werden sollte. Peter deLeon hat denn auch in seiner zusammenfassenden Arbeit aus dem Jahr 1978 (deLeon 1978a, b) das Design der richtigen intrinsischen Anreize der beteiligten Akteure als wichtigsten Schritt zur Entwicklung einer Strategie der Politikbeendigung dargestellt. ${ }^{16}$ Alle diese Instrumente haben sich aber in der Praxis

\footnotetext{
${ }^{16}$ DeLeon dachte an folgende Instrumente: So könnte beispielsweise das Budget einer aufgelösten Politik der betreibenden Behörde zur anderweitigen Verwendung belassen werden; wissenschaftliche Evaluierung von Programmen selbst könnten systematisch intensiviert bzw. die Entscheidung der Weiterführung mit entsprechenden Debatten verknüpft werden (deLeon 1978a: 294 ff.).
} 
als wenig effektiv erwiesen; wohl auch deshalb, weil sich mit diesen Mechanismen die eigentliche politische Auseinandersetzung um einzelne Programme letztendlich nicht ohne weiteres transzendieren lässt. Solche aufklärerischen Instrumente geraten vielmehr selbst regelmäßig in den Sog der Politics, zu dessen Vermeidung sie eigentlich konzipiert wurden (Behn 1977; Daniels 1993, 1997b; Frantz 2002).

\section{Defizite}

Die Beschäftigung mit den Bedingungen, Hinderungsgründen und Auslösungsfaktoren von Politikbeendigung ist eine Debatte unter amerikanischen Politikwissenschaftlern geblieben. Fast keine empirischen und schon gar keine theoretischen Beiträge sind außerhalb der USA entstanden bzw. basieren auf anderen als amerikanischen Daten (deLeon 1997: 2201; Ausnahmen: Greenwood 1997; Harris 1997; deLeon/Hernández-Quezada 2001; Sato 2002).

Zudem wurden vorwiegend Einzelfallstudien vorgelegt, deren Wert für die Theoriebildung entsprechend eingeschränkt ist. Viel zu selten wurde zudem das vergleichende Fallstudiendesign verwandt (Lambright/Sapolsky 1976; Greenwood 1997), so dass hier eines der größten Defizite der Beendigungsforschung liegt. Umgekehrt gilt, dass die konzeptionellen und theoriebildenden Versuche allesamt aus der frühen Periode der Beendigungsforschung stammen (Bardach 1976; Biller 1976; Kaufman 1976; deLeon 1978). Die später entstandenen Arbeiten haben diese Konzepte dann überwiegend nur auf neue Fälle angewandt und somit deren (im Einzelfall größere oder geringere) Relevanz verdeutlicht; und auch die wenigen Artikel mit innovativem Charakter beschränken sich auf die Kritik bzw. die Feinabstimmung einzelner Aspekte (Frantz 1992, 2002; Daniels 1997b; Lewis 2002), ohne auf eine neue Grundlegung des Politikbeendigungsansatzes abzuzielen. Damit sind die theoretischen Linien der Debatte im Großen und Ganzen dort verblieben, wo sie Peter deLeon 1978 in etwa gezogen hat (Greenwood 1997: 2122).

Damit klingt bereits ein weiterer Mangel der Beendigungsliteratur an: praktisch kam es nicht dazu, die gewonnen Erkenntnisse für andere politikwissenschaftliche Debatten fruchtbar zu machen und damit die Relevanz der eigenen Forschung auszuweiten. Zu den Ausnahmen zählen Hogwood und Peters (1982) sowie 
neuerdings Lewis (2002) und Frantz (2002). ${ }^{17}$ Schleierhaft bleibt in diesem Zusammenhang die vollständige Abkopplung der Beendigungsliteratur von dem die Politikwissenschaft seit Mitte der 1980er Jahre immer stärker prägenden Neoinstitutionalismusdiskurs, obwohl die Langlebigkeit sowie Reproduktionsfähigkeit institutioneller Arrangements und deren Persistenz auch angesichts von großem Änderungsdruck deutlich auf gemeinsame Forschungsfragen hindeuten (Hall/Taylor 1996).

Die Politikbeendigungsliteratur hat es zudem versäumt, systematische Ländervergleiche anzustellen. Dies ist im Grunde nur damit zu erklären, dass die einschlägigen Forscher anhand von Daten sowie im Blick auf die innenpolitischen Diskussionen der USA gearbeitet haben. Die vergleichende Perspektive könnte jedoch eine Erfolg versprechende Forschungsstrategie sein, wenn es darum geht, die kausalen Faktoren, die für den Abbau und die Beendigung von Politiken relevant sind, $z u$ isolieren. Ein wesentlicher Ertrag einer erneuerten Politikbeendigungskonzeption müsste daher darin bestehen, die Eigenheiten politischer Systeme und deren Institutionen mit Beendigungsprozesse systematisch vergleichend in Zusammenhang zu bringen (Peters 1998).

Ferner hat es die Beendigungsliteratur nicht geschafft, für zwei zentrale methodische Probleme ihres Gegenstandes befriedigende Lösungen anzubieten. Zum einen bleibt der verwendete Policy-Begriff merkwürdig vage. Wenn Policy Analyse das Studium von Politikinhalten im Kontext von politischen Prozessen und Strukturen ist, erstaunt es um so mehr, dass die Beendigungsforscher nicht systematisch versucht haben, dem Phänomen der Politikbeendigung über eine konsequente Typologisierung von Politikinhalten oder -wirkungen beizukommen. Wo dies ansatzweise geschah, ist der deskriptive wie analytische Erkenntnisgewinn durchaus überzeugend (Lambright/Sapolsky 1976; Kirkpatrick/Lester/Peterson 1999). Zum anderen hat man dem Explanandum, d.h. der abhängigen Variable Politikbeendigung, ohne Not

\footnotetext{
${ }^{17}$ Insbesondere Lewis zeigt, dass die Terminierungsforschung durchaus geeignet ist, Argumente anderer Diskussionen zu hinterfragen. So weisen seine Ergebnisse darauf hin, dass unabhängige Behörden viel öfter als angenommen auch wieder aufgelöst werden, was einige Prämissen der neueren Agencytheorie empfindlich trifft (McCubbins u.a. 1989; Moe 1989; Majone 1996). Diese Erkenntnisse sind allerdings mehr für den Zweit der Organisationsbeendigung von Interesse, der nicht im Mittelpunkt dieser Arbeit steht. Janet E. Frantz versucht dagegen, anhand des konstruktivistisch geprägten „Policy Paradox“-Ansatzes von Deborah Stone die den Beendigungsadvokaten zur Verfügung stehenden politischen Ressourcen neu auszuloten (vgl. Stone 1997).
} 
entscheidende Varianz geraubt, in dem man sich in den Fallstudien überwiegend Beispielen tatsächlicher Beendigung widmete. Man hat auch unkluger Weise darauf verzichtet, mehr intellektuelle Investitionen in heuristische Konzepte für partielle Beendigungen (vgl. deLeon 1978a; Hogwood/Peters 1982) oder für das langsame Austrocknen von Politiken zu tätigen (auch hier wieder mit der Ausnahme Lambright/Sapolsky 1976). Sicher sind die Grenzverläufe zwischen polit-taktischer Rhetorik und ernsthaftem Beendigungsversuch abstrakt nicht einfach zu bestimmen (vgl. Daniels 2001: 252). Im Einzelfall müssten diese aber durchaus empirisch unterscheidbar sein. Wie schwierig auch immer diese Unterscheidung ist, nur mit dem Ausschöpfen der vollen Varianz auf der Seite der abhängigen Variable eröffnet sich eine Chance, zu theoretisch gehaltvollen Aussagen über Kausalbeziehungen im Hinblick auf Politikbeendigung zu kommen.

Schließlich fällt auf, dass der Beendigungsansatz außerhalb der USA praktisch nicht rezipiert worden ist. Weder in Europa generell noch speziell in Deutschland, wo die Policy Analyse ohnehin erst mit einiger Verspätung Fuß zu fassen begann (WindhoffHéritier 1987; Schneider/Janning 2005), hat es eine fokussierte Politikbeendigungsdiskussion gegeben. Selbst auf die in Deutschland unter dem Begriff der „Aufgabenkritik“ geführte Debatte lässt sich kein Einfluss des amerikanischen Beendigungsansatzes ausmachen, obwohl dies thematisch nahe liegen würde. Bei der deutschen Aufgabenkritik steht zudem seit der Übernahme betriebswirtschaftlicher Konzepte im Kontext der New Public Management Welle die Neugewichtung der Beziehung zwischen Staat und Wirtschaft insgesamt im Mittelpunkt (König/Füchtner 2000). Der Aspekt der Politikbeendigung ist dort damit letztlich nur ein nachrangiges Element eines umfassenderen Verwaltungsmodernisierungsprojekts (Fiedler 1998).

\section{Anforderungen an einen erneuerten Politikbeendigungsansatz}

Wird als wünschenswertes Ziel die empirisch fundierte Erneuerung des Politikbeendigungsansatzes akzeptiert, muss für die folgenden konzeptionellen Teilschritte akzeptable Lösungen gefunden werden.

Von entscheidender Bedeutung ist es beispielsweise, die Konzepte Politikbeendigung und Abbau von anderen Formen des Politik- oder 
Organisationswandels abzugrenzen (vgl. Hall 1993; Dunsire/Hood 1989; Tarschys 1985; Levine 1978; Gourevitch 1986; Pierson 1994). Wo verläuft die Grenze zwischen Wandel, Erneuerung und Beendigung? Das Politikarsenal moderner Wohlfahrtsstaaten ist einem ständigen, überwiegend inkrementellen Veränderungsprozess unterworfen (Lindblom 1959; Hogwood/Peters 1985). Wandel und Reform schaffen aber nicht nur Neues. Sie führen in der Regel auch dazu, dass - zumindest - bestimmte Politikinstrumente, Leistungen oder Teilpolitiken nicht mehr eingesetzt bzw. erbracht werden und bestimmte Politikziele oder Politikteilziele nicht mehr weiter verfolgt werden. Die Frage, ob systematisch erklärt werden kann, warum bestimmte politische Teilziele oder Politikinstrumente ihre Bedeutung verloren haben und beendet wurden, wird in der Regel aber gar nicht gestellt (Jann/Wegrich 2003).

Belastbare Generalisierungen über Wirkungszusammenhänge im Beendigungsprozess sind zudem realistischer Weise nur im Vergleich zwischen verschiedenen Politikfeldern (Subventionsabbau, Einsparungen innerhalb Sozialprogrammen, Rücknahme von Regulierungen) und verschiedenen Ländern zu erwarten. Die komparative Vorgehensweise hat den entscheidenden Vorteil, auf die Erfahrungen bzw. vorhandenen Erklärungsmechanismen aus institutionell wie sozioökonomisch unterschiedlicher Länder zurückgreifen zu können. In diesem Kontext scheinen die Erfolgsaussichten am größten, theoretisch abgeleitete Hypothesen auch systematisch testen zu können und im Sinne eines quasiexperimentellen Forschungsdesigns tatsächlich kausale Beziehungen offen zu legen. Hier könnten Standardhypothesen nach der Relevanz sozioökonomischer Entwicklungsniveauunterschieden ebenso abgefragt werden wie die übliche Vetospielervarianz zwischen konsensualen und majoritären politischen Systemen. Darüber hinaus muss die Frage thematisiert werden, wie sich eine Mitgliedschaft in Internationalen Regimen wie der Europäische Union oder der WHO auf nationale Beendigungsprozesse auswirkt.

Ein weiteres Ziel sollte sein, dem Zusammenhang zwischen Politikmisserfolg und der Wahrscheinlichkeit der Politikbeendigung nachzugehen und eine Erklärung dafür zu finden, dass sich Ineffizienz von Politiken häufig nicht negativ auf deren Persistenz auswirkt. Aus der Verwaltungswissenschaft ist das Paradox des Fortbestands von Organisationseinheiten und Politiken bei Aufgabenerledigung bzw. Aufgabenwegfall wohl bekannt (Seibel 1997). Die systematische Erklärung von Persistenz von 
Politiken angesichts offensichtlicher Ineffizienz ist eine weitere Herausforderung und könnte - insbesondere über eine systematische Einbindung von Phänomenen der Nicht-Beendigung angesichts suboptimaler Leistungserbringung - den zu erneuernden Beendigungsansatz entscheidend komplettieren.

Soll die Politikbeendigung jedoch tatsächlich (wieder) als policyanalytisches Konzept fruchtbar gemacht werden, sind die Politikinhalte selbst stärker als bisher in den Vordergrund zu rücken. Unterschiedliche Programmwirkungen (distributiv, redistributiv), angewandte Steuerungsprinzipien (Anreiz- oder Angebotssteuerung), und Charakteristika der Programmbeschaffenheit (materielle Leistungen, immaterielle Leistungen, verhaltensnormierend ohne Leistungscharakter also regulativ) müssen systematisch unter dem Blickwinkel ihrer spezifischen Rückwirkungen auf etwaige Terminierungsprozesse erforscht werden. Es ist nur zu wahrscheinlich, dass politische Konflikte um die Abwendung einer Politikbeendigung im Bereich Forschungsförderung, Sozialpolitik oder der Steuergesetzgebung sehr unterschiedlichen Charakter aufweisen und letztlich auch von unterschiedlichen Akteursgruppen geprägt werden. Dieser Herausforderung hat sich die Beendigungsforschung bisher noch in keiner zureichenden Weise angenommen.

Schließlich könnte sich auch die klassische Parteipolitik als wichtige Variable im Kontext der Politikbeendigungsforschung entpuppen (vgl. Schmidt 2003). Wieder ist festzustellen, dass parteipolitischen Wirkungsfaktoren bisher in der Beendigungsdebatte keine systematische Erklärungskraft zugemessen wurde. Hier könnte sich die amerikanische Dominanz in der Literatur erfahrungsverengend ausgewirkt haben, da sich in den Vereinigten Staaten die Kritiker des „Big Government" - insbesondere auf der Ebene der Bundesstaaten, auf der die meisten Fallstudien angesiedelt sind - nicht eindeutig einer parteipolitischen Ideologie zuordnen lassen. Zumindest in Teilen Europas ist das aber anders. Das konservative Lager und das sozialdemokratisch-sozialistische Lager sind oft relativ eindeutig programmatisch festgelegt, ob die Priorität gegebenenfalls auf der bürgerlichen Eigenverantwortung oder auf der Vorsorgepflicht des Staates liegt (vgl. Bräuninger 2004). 


\section{Konturen einer Heuristik zur Erforschung von Politikbeendigungs- phänomenen: Differenzierungen und Hypothesen}

Weder ist es an dieser Stelle möglich die Defizite der Beendigungsforschung allesamt zu beheben, noch können die oben ausgeführten potentiell Erfolg versprechenden Anforderungen an eine neue kohärente policyanalytische Konzeption der Politikbeendigung ad hoc synthetisiert werden. Hierzu bedarf es selbstredend weiterer empirischer und theoretischer Anstrengungen. Vordringlich sind zunächst die weitere Differenzierung des engeren Politikbeendigungsbegriffs und die Skizzierung eines Analyseansatzes, um empirisch testbare Hypothesen aufstellen zu können und auf diese Weise die zu führende Debatte zu systematisieren.

Vorderhand gilt es daher die bisherige Kategorisierung der Politikbeendigung - nach deLeon Programm, Policy, Organisation und Funktion - zu überdenken. Zumal einerseits "Organisation“ in diesem Schema ein Fremdkörper geblieben ist. Andererseits „Funktion“ als essentielle Staatsaufgabe wie Umverteilung, innere Sicherheit oder Verteidigung gedacht wurde (deLeon 1978a; vgl. Abschnitt 2), die wohl kaum auf dieser Ebene „abgebaut“ oder gar beendet werden. Denn dies würde einen umfassenden wie kurz und mittelfristig unwahrscheinlichen Wandel des dahinter stehenden Staatsverständnisses zur Bedingung haben. Eine realistischere Differenzierung könnte also einen feinkörnigeren Zugriff auf das Beendigungsgeschehen ermöglichen. Ich schlage daher vor, zwischen Leistung, Instrument/Leistungsart, Programm, Politikziel und Staatsaufgabe zu unterscheiden.

Leistung verstanden als Leistungshöhe umfasst hier die Festlegung bestimmter Transferzahlungshöhen oder Grenzwerte, die über Subventionsausschüttung bzw. Steuervergünstigungen entscheiden. Wird der Grundbetrag des Arbeitslosengeldes II auf $400 €$ oder $350 €$ festgelegt bzw. ab welcher Einkommenshöhe können bestimmte Abschreibungen oder Unterstützungen nicht mehr in Anspruch genommen werden? Unter einem Instrument ist eine staatliche Sach-, Dienstleistung, ein Finanztransfer oder eine sonstige Regulierungsleistung zu verstehen. Ein Programm bündelt mehrere Instrumente und mehrere Programme fügen sich in der Regel zu einem Politikziel zusammen (Naturschutz, Altersarmutsbekämpfung, Behindertenpolitik, Gleichberechtigung). Diese Politikziele füllen wiederum allgemein akzeptierte bzw. 
auf dieser Abstraktionsebene zumeist konsensuale Staatsaufgaben (Umweltschutz, Generationengerechtigkeit, Solidarität, Chancengleichheit) aus.

Ferner ist die zutreffende Kritik, dass in westlichen Wohlfahrtsstaaten inkrementalistischer Politikwandel vorherrscht und revolutionäre Brüche selten sind (Lindblom 1959; Hogwood/Peters 1982, 1985; Weaver 1988), ernst zu nehmen, aber gleichzeitig für eine Beendigungsperspektive fruchtbar zu machen. Not tut eine Kategorisierung der Intensität des Beendigungsgeschehens. Der Begriff Politikbeendigung sollte dabei als besondere Ausprägung von Politikwandel konzeptualisiert werden. Auf der Ergebnisebene könnte idealtypisch zwischen Status quo, Ersetzung, Abbau und Beseitigung als unterschiedlich intensive Kategorien von Politikbeendigung unterschieden werden (siehe unten). Die Beendigungsintensität und damit der zu erwartenden Widerstand betroffener Akteure würden von teilweiser Ersetzung bis zur vollständigen und ersatzlosen Beseitigung ansteigen. Gleichzeitig kann man im Anschluss an die Unterscheidungen des vorigen Absatzes davon ausgehen, dass Beendigungsentscheidungen betreffend Leistung, Instrument, Programm, Politikziel und Staatsaufgabe den politisch verantwortlichen Akteuren immer schwerer fallen, da die notwendige politische Unterstützung für eine Beendigung in der angegebenen Reihenfolge fortlaufend schwieriger zu generieren sein wird.

\section{Abbildung $1 \quad$ Differenzierung des Konzepts der Politikbeendigung}

\section{Zunahme Klientelwiderstand}

\section{Status Quo Ersetzung Abbau Beseitigung}

Leistung

Instrument

Programm

Politikziel

Staatsaufgabe

Generierung der notwendigen

politischen Unterstützung

für eine Beendigung wird

immer schwieriger 
Von der empirisch fundierten Kritik an der bisherigen Theorie der Politikbeendigung konnte gelernt werden, dass von speziellen Anreizen (z.B. Sunset-Regelungen und Evaluierungen) oder von den Besonderheiten der Kostenstruktur einer bestimmten Politik praktisch keine zielgerichteten Impulse auf Beendigungsprozesse ausgehen (deLeon 1983; Dery 1984). Wertfragen sowie ideologisch vermittelte Perzeptionen von Kausalzusammenhängen spielen die weitaus wichtigeren Rollen. Daneben bleibt der Klientelwiderstand eine zentrale Variable zur Erklärung der Seltenheit des Phänomens Politikbeendigung. Wobei Klientel hier sehr weit zu verstehen ist und alle diejenigen einschließt, die von einer staatlichen Leistung bzw. deren Bereitstellung als Begünstigte, Produzenten oder administrative und politische Advokaten profitieren.

Ich schlage daher vor, unter dem Konstrukt der „politischen Unterstützung“ insbesondere zwei Aspekte zu fassen: Einmal die Einbettung einer politischen Maßnahme in die Wertehierarchie einer Weltanschauungsgemeinschaft (Wertaspekt). Zweitens die - sicherlich ideologisch vermittelte - konsensuale Perzeption der vorherrschenden Kausalzusammenhänge, aus der sich kohärente Anleitungen zur Problemlösung ergeben (Sachaspekt).

So konzeptualisiert lassen sich für zwei entscheidende Fragen erste Hypothesen bilden, nämlich für die Wahl des Beendigungsobjekts sowie für die Wahl der Beendigungsintensität.

Hypothese 1: Mit zunehmender Beendigungsintensität (Abbau, Ersetzung, Beseitigung) steigt der Widerstand der betroffenen Klientel. Akteure, die direkt oder indirekt von einer staatlichen Leistung bzw. deren Bereitstellung profitieren, wehren sich gegen eine drohende Beendigungsentscheidung und machen dagegen politisch mobil.

Hypothese 2: Politische Akteure werden ein Beendigungsobjekt wählen, dessen ideologische Verortung möglichst weit von den Kernwerten derjenigen entfernt ist, auf deren mehrheitliche Unterstützung die Akteure angewiesen sind. Da unspezifizierte Staatsaufgaben und Politikziele zumeist als Konsens breiter politischer Kräfte gelten können, ist deren Beendigung kaum zu erwarten. Tatsächliche Beendigungen werden daher vor allem bei Leistungen, Instrumenten und Programmen auftreten. 
Hypothese 3: Politische Akteure werden - unter Einbeziehung von Hypothese 1 - ein Beendigungsobjekt danach auswählen, ob Unsicherheit oder aber eine eindeutig negative Meinung über die Bewertung der Zielerreichung im Sinne von Effektivität und Effizienz, d.h. was die Kausalwirkung einer bestimmten Maßnahme anbelangt.

Hypothese 4: Politische Akteure werden die angestrebte Beendigungsintensität zudem danach ausrichten, ob die Kostenverteilung bei der betroffenen Politikklientel diffus oder konkret ist. Einer mobilisierungsstarken Politikklientel werden dabei nach Möglichkeit keine oder nur marginale Kosten auferlegt. Je diffuser die Kostenverteilungswirkung einer Beendigungsentscheidung, desto größer wird die Beendigungsintensität ausfallen.

Diese Hypothesen können als „Isolationsthese“ zusammengefasst werden: Das Risiko unter Beendigungsdruck zu geraten, wächst, wenn eine Maßnahme ideologisch wie klientelpolitisch in einen Randbereich gerückt werden kann. Es ist dabei anzunehmen, dass sozioökonomische Faktoren, technischer Fortschritt, unerwartete Ereignisse oder Skandale im Sinne einschlägiger Ansätze der Policy Analyse (Kingdom 1995; Sabatier 1993b) - auf die politische Unterstützung und auf den Klientelwiderstand einwirken und diese Faktoren gegebenenfalls verstärken oder abschwächen. Was die Auswahl von Beendigungsobjekten und die Intensität von Beendigungsprozessen aber entscheidend beeinflusst, ist in wieweit die Einzelmaßnahmen ideologisch, sachpolitisch und klientelökonomisch „eingebettet" und damit geschützt sind. Je irrelevanter ideologische Verortungen und Vereinnahmungen, je umstrittener die kausale Zuordnung positiver und zielführender Effekte, je zersplitterter und zerstreuter also das Feld der Begünstigten und umso so umfassender die Isolierung einer Maßnahme, desto größer die Wahrscheinlichkeit, dass sich Beendigungsbegehrlichkeiten auf sie richten.

Geht man als Antezedenzbedingung davon aus, dass es überhaupt nur dann zu Beendigungshandeln kommen kann, wenn auf der Ebene der perzepierten Kausalzusammenhänge die positive Wirkung einer bestimmten Maßnahme umstritten ist bzw. angezweifelt wird, kann man aus diesen Hypothesen die Variablen Ideologiebezug und Klientelwiderstand berechtigterweise besonders hervorheben und folgende Policy-Typologie erstellen. 
Abbildung 2: Beendigungsrisiko Policy-Typologie ${ }^{18}$

\begin{tabular}{|l|l|l|l|}
\hline \multicolumn{2}{|l|}{} & \multicolumn{2}{l|}{ Ideologiebezug } \\
\cline { 3 - 4 } \multicolumn{2}{|l}{} & Kern & Peripherie \\
\hline \multirow{3}{*}{ Klientelwiderstand } & stark & tabuisierte Policy & gestaltbare Policy \\
\cline { 2 - 4 } & & & \\
\cline { 2 - 4 } & schwach & orthodoxe Policy & verzichtbare Policy \\
\hline
\end{tabular}

Für eine tabuisierte Policy ist die Beendigungswahrscheinlichkeit äußerst gering. Sie ist zu stark im ideologischen Identitätskern einer politischen Gemeinschaft verankert und ferner auch durch das glaubhafte Drohpotential einer mobilisierungsstarken Klientel gesichert. Hier ist aus der Beendigungsperspektive der Erhalt des Status Quo zu erwarten. Die orthodoxe Policy ist zwar im ideologischen Kerngebiet zu verorten, sie verfügt aber nur über limitierte Klientelbeziehungen bzw. ihr Klientel ist mobilisierungsschwach (vgl. Olson 1971). Tiefgreifender Wandel einer orthodoxen Politik ist aber angesichts der ideologischen Absicherung unwahrscheinlich. Es ist zu erwarten, dass Beendigungsdruck hier höchstens zu Abbauprozessen führt. Verbinden sich analytisch die Eigenschaften ideologisch peripher bei starkem Klientelschutz erhält man den Typ einer gestaltbaren Policy; die Charakteristika ideologisch peripher gekreuzt mit schwachem Klientelschutz kann man als verzichtbaren Policy-Typus bezeichnen. Bei einer gestaltbaren Policy ist ein relativ großer politischer Handlungsspielraum vorhanden, der durch Aushandlung mit den Klientelinteressen geformt und genutzt werden kann. Das zu erwartende Beendigungsresultat ist hier die Ersetzung. Schließlich kommt man analytisch zu der Kategorie der verzichtbaren Policy, die bei Beendigungsdruck die Verhandlungsmasse darstellt und deren Verlust im Hinblick auf die Klientelbeziehungen und auf ideologische Rücksichten verschmerzbar ist.

${ }^{18}$ Uusikylä (1996) erstellte zur Charakterisierung der Cutbackfähigkeit von Organisationen mit anderen Variablen (Institutionalisierung, Mobilisierungsfähigkeit) eine ähnliche Typologie. 


\section{Ausblick}

Politikbeendigung macht als heuristisches Konzept nur Sinn, wenn es ermöglicht, bestimmte Phänomene oder Resultate des politischen Konfliktaustrags in einer besonderen, abgrenzbaren Klasse zusammenzufassen und damit einer systematischen Analyse zugänglich zu machen. Mit der vorgeschlagenen Differenzierung des Politikbeendigungsbegriffs auf der Resultatsebene (Abbau, Ersetzung, Beseitigung) sowie auf der Objektebene (Leistung, Instrument, Programm, Politikziel, Staatsaufgabe) scheint ein bescheidener Schritt zur Weiterentwicklung des Konzepts getan. Es ist dabei nicht gesagt, dass politische Prozesse im Beendigungsbereich wesentlich anders ablaufen als in den anderen Phasen des Politikzyklus. Aus der Perspektive der politischen Steuerung bleibt aber zu klären, welches die Erfolgs-und Misserfolgsbedingungen für absichtsvolles politisches Handeln sind, das den Abbau, die Ersetzung zum Zwecke der teilweisen Reduktion oder die gänzliche Beseitigung von öffentlichen Maßnahmen verstanden als Leistungen, Instrumente, Programme, Politikziele und Staatsaufgaben zum Ziel hat. Die Isolationsthese, entlang derer Politikbeendigungsphänomene mit einem Fokus auf Aspekte der Klientelstruktur und der Weltanschauung erklärt werden können und die die Grundlage für die vorgestellte Typologie bildet (vgl. Abschnitt 6), ist eine denkbare Argumentationslinie wie der Untersuchungsgegenstand Politikbeendigung im Hinblick auf Steuerungsfragen systematisiert und für eine empirische Analyse fruchtbar gemacht werden kann. Daneben gibt es selbstredend noch eine Reihe anderer interessanter Gesichtspunkte, von denen beispielsweise die erwähnten institutionellen Hypothesen einigen Erklärungsmehrwert versprechen (vgl. Abschnitt 5). Denkt man die Argumente zur Klientelstruktur weiter, rücken zudem insbesondere klassische Systemunterschiede wie die korporatistische versus pluralistische Interessenaggregation und deren Einfluss auf nationale Beendigungsprozesse als lohnenswerte Forschungsfragen in den Blick.

Schließlich hängt die Bedeutsamkeit eines erneuerten Beendigungsansatzes aber auch davon ab, ob in der Realwelt Beendigungs- und Abbauphänomene tatsächlich von Relevanz sind. Faktisch finden sich derzeit viele Beispiele, die sich - sei es als politische Forderung im Sinne eines Für und Wider, sei es als tatsächliche Entscheidung - vorderhand unter Beendigungskategorien subsumieren lassen. So wurde die Eigenheimzulage als Wohneigentumförderinstrument des Bundes ebenso 
abgeschafft wie in Niedersachsen das Landesblindengeld. In Hessen wurde die Schuldnerberatung oder in Bayern die präventive Jugendarbeit empfindlich gekürzt. ${ }^{19}$ Verschiedene Steuerprivilegien - für Lebensversicherungen bis hin zur Haltung von Geländewagen - wurden jüngst ebenfalls gestrichen. Zudem zeichnet sich ab, dass bei verschiedenen Verwaltungsstrukturreformen in den Ländern zumindest billigend in Kauf genommen wird, dass ehemalige Umweltschutzniveaus und Naturschutzplanungskapazitäten nicht mehr erreicht werden können. ${ }^{20}$

In der politischen Debatte scheinen sich zudem die Gewichte zu verschieben. Dem „mutigen Sparen“ und dem „Subventionsabbau“ wird „Vorrang“ eingeräumt (Koalitionsvertrag 2005). Staatsverschuldung, demographischer Wandel und der Veränderungsdruck der Globalisierung werden in diesem Kontext immer wieder als Faktoren angeführt, die den Druck auf den Wohlfahrtsstaates erhöhen (BertelsmannStiftung 2005). Der Staat müsse seine Ausgaben und damit seine Aufgaben drastisch einschränken. Ohne eine nachhaltige Sanierung des Staatshaushaltes, droht in Kürze, so das Hauptargument, der Verlust der politischen Steuerungsfähigkeit des Gemeinwesens - so oder so ähnlich die Kassandrarufe. ${ }^{21}$ Mit der Initiative für Bürokratieabbau der alten Bundesregierung, den verschiedenen „better regulation“ Initiativen auf europäischer und internationaler Ebene und Streichvorstößen verschiedener Politiker waren politische Abbauaktivitäten bereits vor dem Regierungswechsel 2005 präsent. ${ }^{22}$ Seit dem Regierungsantritt der Schwarz-Roten Koalition hat sich diese Tendenz verstetigt: so wird bei der Pendlerpauschale und beim Sparerfreibetrag entscheidend gekürzt. Ferner sollen Kursgewinne bei Aktienverkäufen und Gewinne aus Immobiliengeschäften künftig generell steuerpflichtig werden. Steuerberatungskosten können künftig nicht mehr von der Steuer abgesetzt werden ebenso sollen Abzugsmöglichkeiten für

\footnotetext{
$19 \mathrm{Vgl}$. http://www.bjr.de/presse/pressemitteilungen/uebersicht-2004.php?pmid=25\&link= uebersicht2004, http://www.spd-fraktion-hessen.de/site/Streichliste.160.0.html und http://www.goest.de/sozialabbau2004.htm (letzter Zugriff 22.12.2005).

${ }^{20}$ Vgl. interuniversitäre Forschungsprojekt Knill, Christoph/Bogumil, Jörg/Bauer, Michael W. zur Modernisierung der Umweltverwaltung unter http://www.unikonstanz.de/FuF/Verwiss/knill/projekte/ moduv/moduv-d.html, letzter Zugriff 22.12.2005. Der Umstand, dass es in jüngster Zeit mehr Politikbeendigungen zu geben scheint, könnte allerdings auch auf einem Wahrnehmungsbias beruhen. Es ist letztlich eine empirisch zu klärende Frage, ob es nicht auch in früheren Zeiten staatsbudgetärer Notlagen zu (mehr) Politikabbau, Ersetzung oder Beseitigung gekommen ist.

${ }^{21}$ So wird der Präsident des Bundesverfassungsgerichts in der F.A.Z. vom 27. Dezember 2004, Seite 4 , zitiert.
} 
Schulgeldzahlungen an Privatschulen wegfallen. Auch die Freibeträge für Abfindungen bei Entlassungen sowie die Bergmannsprämie sollen gestrichen werden und die private Nutzung von Dienstwagen wird höher besteuert werden. ${ }^{23}$ Diese Auflistung erklärt an sich noch nichts. Die Beispiele machen aber plausibel, dass Politikabbau, -ersetzung und -beseitigung Phänomene sind, denen im gegenwärtigen Politikgeschehen erhöhte Bedeutung zukommt.

Ob die Beendigungsforschung zu Recht der "neglected butt“ (Behn 1978a: 413) der Policy Analyse ist und besser bleiben sollte, kann und will dieser Artikel nicht entscheiden. Angesichts immer intensiveren Diskussionen über eine notwendige Aufgabenreduktion der westlichen Wohlfahrtsstaaten - zumindest kontinentaleuropäischer Provenienz - und klarer Evidenz für öffentliches Abbauhandeln scheint es aber gerechtfertigt und geboten, die Frage nach einem politikwissenschaftlichen Beitrag zum Verständnis von Beendigungsprozessen neu zu stellen.

\footnotetext{
${ }^{22}$ Wie zuletzt der Ankündigung von Kommissionspräsident José Manuel Brroso, die EU ziehe 68 geplante (!) Gesetzevorlagen zurück (vgl. http://europa.eu.int/newsletter/archives2005/issue68/ index_en.htm, letzter Zugriff 22.12.2005).

${ }^{23} \mathrm{Vgl}$. 'Im neuen Jahr treffen erste Sparmaßnahmen von Schwarz-Rot die Bürger', in: FAZ vom 31.12.2005, Seite 18.
} 


\section{Literaturverzeichnis}

Bardach, Eugene, 1976: Policy Termination as a Political Process, in: Policy Sciences 7, 123-131.

Behn, Robert D., 1976: Closing the Massachusetts Public Training Schools, in: Policy Sciences 7, 151-171.

Behn, Robert D., 1977: The False Dawn of Sunset Laws, in: Public Interest 49, 103-118.

Behn, Robert D., 1978a: How to Terminate a Public Policy: A Dozen Hints for the Would-be Terminator, in: Policy Analysis 4, 393-414.

Behn, Robert D., 1978b: Closing a Government Facility, in: Public Administration Review 38, 332-338.

BertelsmannStfitung, 2005: Schuldenmonitor, Gütersloh.

Biller, Robert P., 1976: On Tolerating Policy and Organizational Termination: Some Design Considerations, in: Policy Sciences 7, 133-149.

Bothun, Douglas/Comer, John C., 1979: The Politics of Termination: Concepts and Process, in: Policy Studies Journal, 540-553.

Bradley, Valerie J., 1976: Policy Termination in Mental Health: The Hidden Agenda, in: Policy Sciences 7, 215-224.

Bräuninger, Thomas, 2004: A Partian Model of Government Expenditure, in: Public Choice, 30, 1: 121.

Brewer, Garry D., 1974: The Policy Sciences Emerge: To Nurture and Structure a Discipline, in: Policy Sciences 5, 239-245.

Brewer, Garry D., 1978: Termination: Hard Choices, Harder Questions, in: Public Administration Review 38, 338-344.

Cameron, James M., 1978: Ideology and Policy Termination: Restructuring California's Mental Health System, in: May, Judith V./Wildavsky, Aaron B. (Hrsg.): The Policy Cycle, Beverly Hills: Sage, 301- 328.

Carpenter, Daniel P., 2000: Stochastic Prediction and Estimation of Nonlinear Political Durations: An Application to the Lifetime of Bureaus, in: Richards, Diana (Hrsg.): Political Complexity: Nonlinear Models of Politics, Ann Abor: University of Michigan Press.

Carroll, Glenn R., 1983: A Stochastic Model of Organizational Mortality: Review and Reanalysis, in: Social Science Research 12, 303-329.

Carroll, Glenn R., 1984: Organizational Ecology, in: Annual Review of Sociology 10, 71-93.

Casstevens, Thomas W., 1980: Birth and Death Processes of Government Bureaus in the United States, in: Behavioral Science 25, 161-165.

Casstevens, Thomas W., 1984: Population Dynamics of Governmental Bureaus, in: UMAP Journal 5, 178-199.

Corder, J. Kevin, 2004: Are Federal Programs Immortal? Estimating the Hazard of Program Termination, in: American Politics Research 32, 3-28.

Daniels, Mark R., 1993: Termination, Innovation and the American States: Testing Sunset Legislation, in: American Review of Politics 15, 507-518.

Daniels, Mark R., 1994: Termination and Bureaucracy: Ending Government Programs, Policies and Organizations, in: Farazmand, Ali (Hrsg.): Handbook of Bureaucracy, New York: Marcel Dekker, 446- 466.

Daniels, Mark R., 1995: Organizational Termination and Policy Continuation: Closing the Oklahoma Public Training Schools, in: Policy Sciences 28, 301-316.

Daniels, Mark R., 1996: Implementing Policy Termination: Health Care Reform in Tennessee, in: Policy Studies Review 14, 353-374.

Daniels, Mark R., 1997a: Theories of the Termination of Public Programs, Policies and Organizations, in: International Journal of Public Administration 20, 2043-2066.

Daniels, Mark. R., 1997b: Terminating Public Programs: An American Political Paradox, New York: 
ME Sharpe.

Daniels, Mark. R., 2001: Policy and Organizational Termination, in: International Journal of Public Administration, 24, 249-262.

deLeon, Peter, 1978a: A Theory of Policy Termination, in: May, Judith V./Wildavsky, Aaron B. (Hrsg.): The Policy Cycle, Beverly Hills: Sage, 279-300.

deLeon, Peter, 1978b: Public Policy Termination: An End and a Beginning, in: Policy Analyses 4, 369_ 392.

deLeon, Peter, 1982: New Perspectives on Program Termination, in: Journal of Policy Analysis and Management, 2, 108-111.

deLeon, Peter, 1983: Policy Evaluation and Program Termination, in: Policy Studies Review 2, 631647.

deLeon, Peter, 1987: Policy Termination as a Political Phenomenon, in: Palumbo, Dennis (Hrsg.): The Politics of Program Evaluation, Newbury Park: Sage, 173-199.

deLeon, Peter, 1997: Afterward: The Once and Future State of Policy Termination, in: International Journal of Public Administration, 20, 2195-2212.

deLeon, Peter/Hernández-Quezada, José Mario, 2001: The Case of the National Solidarity Program in Mexico: A Study in Comparative Public Policy Termination, in: International Journal of Public Administration, 24, 289-309.

Dery, D. (1984). "Evaluation and Termination in the Policy Cycle." Policy Sciences 17(1): 13-26.

Dieckmann, Rudolf, 1977: Aufgabenkritik in der Großstadtverwaltung, Berlin.

Ellis, Carol L., 1983: Program Termination: A Word of the Wise, in: Public Administration Review 7 , 352-257.

Fiedler, Jobst, 1998: Aufgabenkritik und Konzentration auf Kernaufgaben, in: Bandemer, Stephan von/Blanke, Bernhard/Nullmeier, Frank/Wewer, Göttrik (Hrsg.): Handbuch zur Verwaltungsreform, Opladen, 93-106.

Foster, James L./Brewer, Garry D., 1976: And the Clocks Were Striking Thirteen: The Termination of War, in: Policy Sciences 7, 225-243.

Frantz, Janet E., 1992: Reviving and Revisiting a Termination Model, in: Policy Sciences 25, 175-186.

Frantz, Janet E., 1997: The High Cost of Policy Termination, in: International Journal of Public Administration 20, 2097-2119.

Frantz, Janet E., 2002: Political Resources for Policy Terminators, in: Policy Studies Journal 30, 1128.

Frantz, Janet E., 2003: The High Cost of Policy Termination, in: Rabin, Jack/Munzenrider, Robert F./Bartell, Sherrie M. (Hrsg.): Principles and Practices of Public Administration, Part 06: Decision Making, Policy Studies and Analysis, ebook, Marcel Dekker, New York.

Geva-May, Iris, 2001: When the Motto is "Till Death do us Part": The Conceptualization and the Craft of Termination in the Public Policy Cycle, in: International Journal of Public Administration 24, 263-288.

Geva-May, Iris, 2004: Riding the Wave Opportunity: Termination in Public Policy, in: Journal of Public Administration Research and Theory 14, 309-333.

Gourevitch, Peter, 1986: Politics in Hard Times. Comparative Responses to International Economic Crises, Ithaca and London: Cornell University Press.

Grafton, Carl, 1984: Response to Change: Creation and Reorganization of Federal Agencies, in: Miewald, Robert/Steinman, Michael (Hrsg.): Problems in Administrative Reform, Chicago: Nelson-Hall.

Greenwood, Justin, 1997: The Succession of Policy Termination, in: International Journal of Public Administration 20, 2121-2150.

Hall, Peter A., 1993: Policy Paradigms, Social Learning, and the State. The Case of Economic Policymaking in Britain, in: Comparative Politics 25, 275-296. 
Hall, Peter A./Taylor, Rosemary C. R., 1996: Political Science and the Three New Institutionalisms, in: Political Studies 44, 936-957.

Hannan, M.T./Freeman, J.H., 1977: The Population Ecology of Organizations, in: American Journal of Sociology, 82, 929-964.

Hargrove, E. C., 1975: The Missing Link: The Study of the Implementation of Social Policy, Washington, D.C.: Urban Institute.

Harris, Michael, 1997: Policy Termination: Uncovering the Ideological Dimension, in: International Journal of Public Administration 20, 2151-2175.

Harris, Michael, 2001: Policy Termination: The Case of Term Limits in Michigan, in: International Journal of Public Administration 24, 323-339.

Héritier, Adrienne, 1993: Policy-Analyse. Elemente der Kritik und Perspektiven der Neuorientierung, in: Héritier, Adrienne, (Hrsg.): Policy-Analyse. Kritik und Neuorientierung, PVS-Sonderheft, Opladen, 9-36.

Héritier, Adrienne/Knill, Christoph/Douillet, Anne-Cécile/Kerwer, Dieter/Lehmkuhl, Dirk/Teutsch, Michael, 2001: Differential Europe. The EU Impact on National Policy Making, Lanham: Rowman \& Littlefield.

Héritier, Adrienne/Knill, Christoph/Mingers, Susanne/Becka, Martina, 1994: Die Veränderung von Staatlichkeit in Europa. Ein regulativer Wettbewerb: Deutschland, Großbritannien und Frankreich in der EU. Opladen: Leske \& Budrich.

Hill, Hermann, 2004: Aufgabenkritik, Privatisierung und Neue Verwaltungssteuerung, Baden-Baden: Nomos.

Hogwood, Brian W., 1996: Changes in Regulatory Agencies in British Government, paper presented at the Conference on Regulatory Organizations, University of Exeter.

Hogwood, Brian W./Peters, Guy B., 1982: The Dynamics of Policy Change: Policy Succession, in: Policy Sciences 14, 225-245.

Hogwood, Brian W./Peters, Guy B., 1985: The Pathology of Public Policy, Clarendon Press, Oxford.

Hooghe, Liesbet, 1996: Cohesion Policy and European Integration: Building Multi-Level Governance, Oxford: Oxford University Press.

Iklé, Fred C., 1976: The Prevention of Nuclear War in a World of Uncertainty, in: Policy Sciences 7 , 245-250.

Jann, Werner/Wegrich, Kai, 2003: Phasenmodelle und Politikprozesse: Der Policy Cycle, in: Schubert, Klaus/Bandelow, Nils C., Lehrbuch der Politikfeldanalyse, Oldenburg, 71-104.

Katzenbach, Edward L., 1958: The Horse Calvary in the Twentieth Century: A Study of Policy Response, in: Public Policy 8, 120-149.

Kaufman, Herbert, 1976: Are Government Organizations Immortal? Washington, D.C.: The Brookings Institution.

Kaufman, Herbert, 1987: Time, Change and Organizations: Natural Selection in a Perilous Environment, Chatham New York: Chatham House.

Kimberly, John R./Miles, Raymond H., 1980: The Organizational Life Cycle: Issues in the Creation, Transformation and Decline of Organizations, San Francisco, CA: Jossey-Bass.

Kingdon, John W., 1995: Agendas, Alternatives and Public Policies, New York. Kirkpatrick, Susan E./Lester, James P./Peterson, Mark R., 1999: The Policy Termination Process: A Conceptual Framework and Application to Revenue Sharing, in: Policy Studies Review 16, 209-236.

Knill, Christoph, 2003: Europäische Umweltpolitik. Steuerungsprobleme und Regulierungsmuster im Mehrebenensystem, Opladen: Leske \& Budrich.

Koalitionsvertrag CDU/CSU, SPD, 2005: Koalitionsvertrag zwischen CDU, CSU und SPD. Gemeinsam für Deutschland - Mit Mut und Menschlichkeit, http://www.cducsu.de/upload/koalitionsvertrag/. 
König, Klaus/Füchtner, Natascha, 2000: Schlanker Staat - eine Agenda für die Verwaltungsmodernisierung im Bund, Baden-Baden: Nomos.

Kotz, Nick, 1988: Wild Blue Yonder: Money, Politics, and the B-1 Bomber, Princeton: Princeton University Press.

Kuipers, S./Boin, A. et al., 2005. "The Life and Death of Public Organizations: Revisiting the New Deal Agencies.", unpublished manuscript, University of Leiden.

Lambright, Henry W./Sapolsky, Harvey, M. 1976, Terminating Federal Research and Development Programs, in: Policy Sciences 7, 199-213.

Lasswell, Harold D., 1951: The Policy Orientation, in: Lerner Daniel/Lasswell, Harold D. (Hrsg.): The Policy Sciences, Stanford, California: Stanford University Press, 3-15.

Lasswell, Harold D., 1971: A Pre-View of Policy Sciences, New York: American Elsevier.

Levine, Charles H., 1978: Organizational Decline and Cutback Management, in: Public Administration Review 38, 316-325.

Lewis, David E., 2002: The Politics of Agency Termination: Confronting the Myth of Agency Immortality, Journal of Politics 64, 89-107.

Lindblom, Charles, 1959, The Science of Muddling Through, in: Public Administration Review, Volume 19, No. 2 (Spring, 1959), pp. 79-88.

Mahoney, James/Goertz, Gary, 2004: The Possibility Principle: Choosing Negative Cases in Comparative Research, in: American Political Science Review 98, 653-669.

Majone, Giandomenico, 1996: Regulating Europe, London: Routledge.

Mayntz, Renate (Hrsg.), 1983 : Implementation politischer Programme II. Ansätze zur Theoriebildung, Opladen: Westdeutscher Verlag.

McCubbins, Mathew D./Noll, Roger/Weingast, Barry, 1989: Structure and Process, Politics and Policy: Administrative Arrangements and the Political Control of Agencies, in: Virginia Law Review 75, 431- 482.

Moe, Terry M., 1989: The Politics of Bureaucratic Structure, in: Chubb, John E./Peterson, Paul E. (Hrsg.): Can the Government Govern?, Washington, D.C.: The Brookings Institution, 267329.

Mueller, Keith J., 1988: Federal Programs to Expire: The Case of Health Planning, in: Public Administration Review 48, 719-725.

Norris-Tirrell, Dorothy, 1997: Organization Termination in the Nonprofit Setting: The Dissolution of Children's Rehabilitation Services, in: International Journal of Public Administration 20, 21772195.

Norris-Tirrell, Dorothy, 2001: Organization Termination or Evolution: Mergers in the Nonprofit Sector, in: International Journal of Public Administration 24, 311-322.

Olson, Mancur, 1971: The Logic of Collective Action: Public Goods and the Theory of Groups, Harvard University Press, Cambridge, Mass.

Peters, B. Guy, 1998: Comparative Politics. Theory and Methods, Houndmills: Palgrave.

Peters, B. Guy/Hogwood, Brian W., 1988: Births, Deaths and Marriages: Organizational Change in the U.S. Federal Bureaucracy, in: American Journal of Public Administration 18, 119-133.

Peters, B. Guy/Hogwood, Brian W., 1991: Applying Population Ecology Models to Public Organizations, in: Research in Public Administration 1, Westport, CT: JAI Press, 79-108.

Pierson, Paul, 1994: Dismantling the Welfare State? Reagan, Thatcher and the Politics of Retrenchment, Cambridge.

Pressman, Jeffrey L.Mildavsky, Aaron B., 1973: Implementation: How Great Expectations in Washington are Dashed in Oakland; or, why it's Amazing that Federal Programs Work at all..., Berkeley: UCP.

Sabatier, Paul A., 1993a: Top-Down and Bottom-Up Approaches to Implementation Research, in: Hill, Michael (Hrsg.): The Public Policy Process: A Reader, London, 266-293. 
Sabatier, Paul A. 1993b: Advocacy-Koalitionen, Policy-Wandel und Policy-Lernen: Eine Alternative zur Phasenheuristik, in: Héritier, Adrienne (Hrsg.): Policy Analyse. Kritik und Neuorientierung, Opladen, Westdeutscher Verlag, 116-148.

Sato, H., 2002: Abolition of Leprosy Isolation Policy in Japan: Policy Termination through Leadership. Policy Studies Journal 30(1): 29-46.

Sato, H. and J. E. Frantz, 2005: Termination of the leprosy isolation policy in the US and Japan: Science, policy changes, and the garbage can model. BMC, International Health and Human Rights 5(3).

Schmidt, Manfred G., 2003: Vergleichende Policy-Forschung, in: Berg-Schlosser, Dirk/Müller-Rommel, Ferdinand (Hrsg.): Vergleichende Politikwissenschaft, 4. Aufl., Opladen: Leske \& Budrich, 261-276.

Seibel, Wolfgang, 1997: Die Treuhandanstalt - Eine Studie über Hyperstabilität, in: Wollmann, Hellmut/Derlien, Hans-Ulrich/König, Klaus/Renzsch, Wolfgang/Seibel, Wolfgang (Hrsg.): Transformation der politisch-administrativen Strukturen in Ostdeutschland, Opladen: Leske \& Budrich, 169-222.

Shulsky, Abram N., 1976: Abolishing the District of Columbia Motorcycle Squad, in: Policy Sciences 7, 183-197.

Stone, Deborah, 1997: Policy Paradox: The Art of Political Decision Making, New York: Norton.

Tarschys, Daniel, 1983: The Scissors Crisis in Public Finance, in: Policy Sciences 15, 205-224.

Tarschys, Daniel, 1985: Curbing Public Expenditure: Current Trends, in: Journal of Public Policy 5, 23-67.

Thai, Khi V./Sullivan, David, 1989: Impact of Termination of General Revenue Sharing on New England Local Government Finance, in: Public Administration Review 49, 61-67.

Uusikylä, P. 1996: The Politics of Cutback Management: A Case Study of Interorganizational Relations and Influence Structures During a Local Government Retrenchment Process. MPIfG Discussion Paper 96/2; http://www.mpi-fg-koeln.mpg.de/pu/dp94-96_de.html.

Wallerstein, Mitchell B., 1976: Terminating Entitlements: Veterans Disability Benefits in the Depression, in: Policy Sciences 7, 173-182.

Weaver, R. Kent, 1988: Automatic Government: The Politics of Indexation, Washington D.C.: The Brookings Institution.

Whetten, David A., 1987: Organization Growth and Decline, in: Annual Review of Sociology 13, 335358.

Windhoff-Héritier, Adrienne, 1987: Policy-Analyse. Eine Einführung, Frankfurt am Main: Campus Verlag.

Wollmann, Hellmut, 2003: Kontrolle in Politik und Verwaltung: Evaluation, Controlling und Wissensnutzung, in: Schubert, Klaus/Bandelow Nils C. (Hrsg.): Lehrbuch der Politikfeldanalyse, 335-360. 


\section{Contact}

Prof. Dr. Christoph Knill

Chair of Comparative Public Policy and Administration

Box D 91

D-78457 Konstanz

Germany

Phone ++497531885597

Fax ++ 497531882381

christoph.knill@uni-konstanz.de

University of Konstanz

(7) www.uni-konstanz.de

Department of Politics and Management

(7) www.uni-konstanz.de/sektionen/polver

Chair of Comparative Public Policy and Administration

(7) www.uni-konstanz.de/FuF/Verwiss/knill

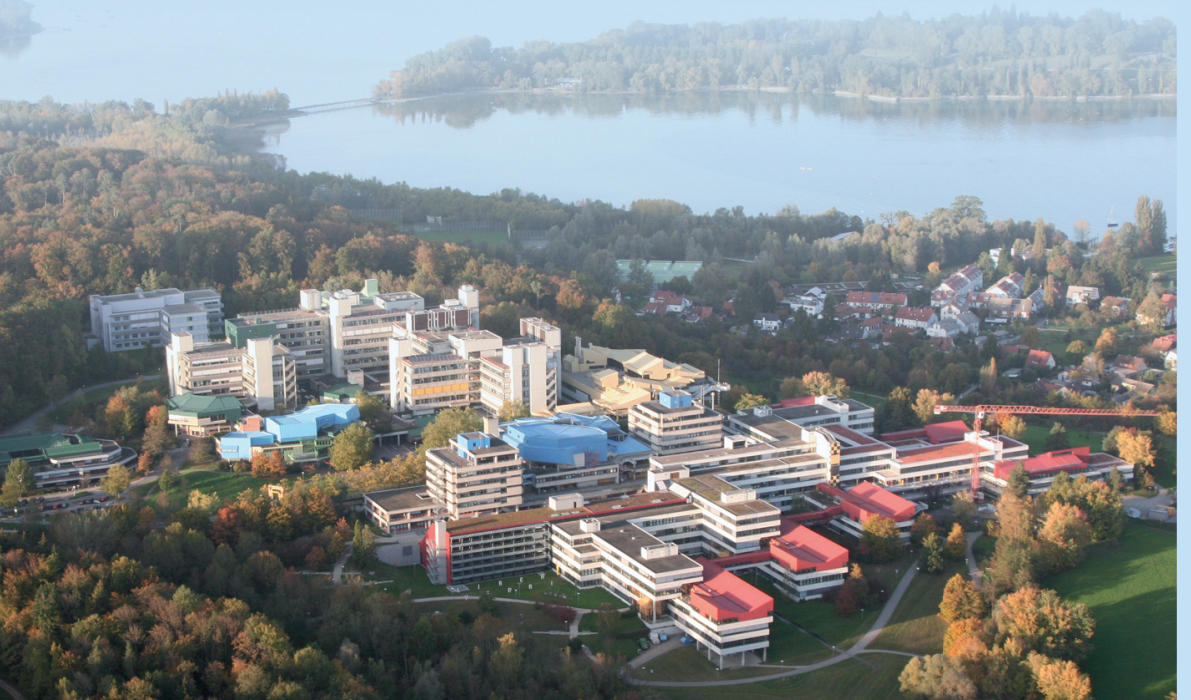

